Open Scientist Handbook • OSH

\title{
The Logic of Abundance in a Gift Economy
}

Bruce R. Caron

Published on: Mar 10, 2021

DOI: $10.21428 / 8 b b b 7 f 85.12 f d b a b c$

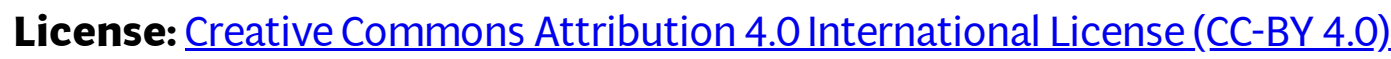






How big is your bucket? Make it smaller to find "enough".

\section{Get Ready for Abundance}

"The opposite of scarcity is not abundance. The opposite of scarcity is... enough"

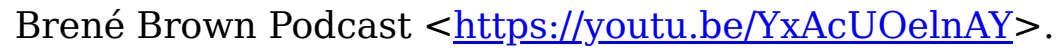

"Scarcity is easier to deal with than abundance, because when something becomes rare, we simply think it more valuable than it was before, a conceptually easy change. Abundance is different: its advent means we can start treating previously valuable things as if they were cheap enough to waste, which is to say cheap enough to experiment with. Because abundance can remove the trade-offs we're used to, it can be disorienting to the people who've grown up with scarcity. When a resource is scarce, the people who manage it often regard it as valuable in itself, without stopping to consider how much of the value is tied to its scarcity" (Shirky 2010).

"For the Ju/'hoansi, that fundamental axiom of modern economics, "the problem of scarcity", simply did not apply. Where this holds that it is human nature to have 
infinite wants and limited means, the Ju/'hoansi had few wants that were simply met" (Suzman 2017 [ The Guardian]).

\section{Abundances}

We have not really begun to explore the many varieties of abundance that can emerge once we abandon arbitrary scarcity. It is important to remember, as Brené Brown (2012) reminds us, that abundance is also simply enough: enough access and discovery tools to know your research is new and where it sockets into existing knowledge; enough communication with colleagues working in your specific arena to collaborate your way through pain points; enough research funding to move ahead; enough time to do your work, and; enough credit to know your work is out there and appreciated. Enough is never too much. It is all the abundance an open scientist needs.

Primary abundance is built into digital science objects which, like Jefferson's thoughts, can be copied infinitely without diminishing the original. Quite the opposite, the more copies that circulate, the more valuable the original object becomes, only not as the private property of an individual, but rather as a common pool resource for the commons.

Combinatory abundance is what happens when science objects (and scientists) enter into collaborative matrix to mix, meld, and produce new objects. This is also where the network effect applies to objects, not just people.

"The difference between humans and animals lies in the ability to collaborate, engage in business, let ideas, pardon the expression, copulate. Collaboration has explosive upside, what is mathematically called a superadditive function, i.e., one plus one equals more than two, and one plus one plus one equals much, much more than three. That is pure nonlinearity with explosive benefits-we will get into details on how it benefits from the philosopher's stone." (Taleb 2012) paraphrasing Ridley (2010).

Language is a good example of the kind of combinatory abundance that open science hopes to achieve through mineable/mixable repositories of a wide variety of knowledge objects. The English alphabet has twenty-six letters and the English language about forty phonemes. From these all words, sentences, paragraphs, texts, and conversations are spun by combining and assembling them using rules and shared semantics. 
You're an academic, you know that academics might run out of patience, or time, or even wine, but rarely do we run out of words (or ideas for that matter). In fact this is one abundance that we have always enjoyed, perhaps a bit too much. To achieve the "explosive upside" of collaboration, scientists need to build open cultures of collaboration.

Emergent abundance describes the complex objects of study, the unknowns that feed science and also science's willingness to not seek "truth". Whether you are tracking the micro-second changes of a single cell or the collision courses of galaxies, you begin with a never-decreasing abundance of questions. Science also has an abundance of doubts, as well as discoveries. Science swims in an ocean (an abundance also) of doubt, as Richard Feynman reminds us: "A scientist is never certain. We all know that. We know that all our statements are approximate statements with different degrees of certainty; that when a statement is made, the question is not whether it is true or false but rather how likely it is to be true or false" (Feynman 2005).

What emerges from these doubts is a collective form of being only slightly less... wrong. Being less wrong iterates into being somewhat more right, but never to the point of actual truth. Everything we know today will be different from what we know tomorrow.

"[S]cientists gravitate toward falsification; as a community if not as individuals, they seek to disprove their beliefs. Thus, the defining feature of a hypothesis is that it has the potential to be proven wrong (which is why it must be both testable and tested), and the defining feature of a theory is that it hasn't been proven wrong yet. But the important part is that it can be - no matter how much evidence appears to confirm it, no matter how many experts endorse it, no matter how much popular support it enjoys. In fact, not only can any given theory be proven wrong; ... sooner or later, it probably will be. And when it is, the occasion will mark the success of science, not its failure" (Schultz 2011).

Infinite abundance marks the recognition that science is not a finite game. There is no way to "win" science; no ending of science; and no possibility for its rules to be fully known; these are continually subject to change. The great mistake of bringing the logic of the marketplace (a finite, zero-sum game) into the academy is that it promotes behaviors that treat science like a finite game, and it makes competitors out of colleagues. 
As a form of infinite play, science finds itself in a never-ending tussle with its objects of study; "Our freedom in relation to nature is not the freedom to change nature; it is not the possession of power over natural phenomena. It is the freedom to change ourselves. We are perfectly free to design a culture that will turn on the awareness that vitality cannot be given but only found, that the given patterns of spontaneity in nature are not only to be respected, but to be celebrated" (Carse 1987). James Carse's book, Finite and Infinite Games, offers a great heuristic for the type of culture change needed for science to become "open science"

"THERE ARE at least two kinds of games. One could be called finite, the other infinite. A finite game is played for the purpose of winning, an infinite game for the purpose of continuing the play."

..."It is on this point that we find the most critical distinction between finite and infinite play: The rules of an infinite game must change in the course of play. The rules are changed when the players of an infinite game agree that the play is imperiled by a finite outcome-that is, by the victory of some players and the defeat of others. The rules of an infinite game are changed to prevent anyone from winning the game and to bring as many persons as possible into the play." (Carse 2011)

Sufficient abundance reminds us that abundance does not need to be a waterfall into an overflowing bucket. As long as the bucket is full, there is abundance. A single extra drop makes it overflow. Abundance is relative to needs, and needs can be managed to the level of sufficiency, rather than expanded by market-fueled desires. In reality, abundance just means this: enough.

Open science advocates are often asked about how they will replace (perverse) market incentives; as if these are the only incentives out there. Scientists have their own incentives, the reasons they are scientists and not, say, hedge fund managers. And scientists were fully incentivized in the decades before the marketplace intruded on the academy. There are many articles about the mismatch between science and market incentives. A good place to start is Edwards and Roy (2016):

"In this article, we will (1) describe how perverse incentives and hypercompetition are altering academic behavior of researchers and universities, reducing scientific progress and increasing unethical actions, (2) propose a conceptual model that describes how emphasis on quantity versus quality can adversely affect true scientific progress, (3) consider ramifications of this environment on the next 
generation of Science, Technology, Engineering and Mathematics (STEM) researchers, public perception, and the future of science itself, and finally, (4) offer recommendations that could help our scientific institutions increase productivity and maintain public trust. We hope to begin a conversation among all stakeholders who acknowledge perverse incentives throughout academia, consider changes to increase scientific progress, and uphold "high ethical standards" in the profession..."

Offer a scientist more time, cheaper tools, and some security to finish their research, and you will have a happy scientist. Chasing reputation points and writing endless proposals for funding would not compete with simply clearing the decks and letting research come to the fore. Managing needs can be a productive alternative to bulking up the CV with marginal publications. Open science can wean the scientist from perverse incentives by offering more with less.

Are you tired of working so hard to get just a bit more? One of the tasks of open science is to innovate to lower the costs of doing science. The most "successful" societies in the history of humanity became affluent by managing their needs:

"[Marshall] Sahlins characterized hunter-gatherers as the gurus of a "Zen road to affluence" through which they were able to enjoy "unparalleled material plentywith a low standard of living." Here, it seemed, was a people unconcerned with material wealth, living in harmony with their natural environments, who were also egalitarian, uncomplicated, and fundamentally free” (Suzman 2017).

Sometimes one can achieve abundance by simply finding a smaller bucket. Matching needs to resources (instead of the other way) can tip your situation from scarcity to "just enough".

\section{The next steady-state of science is the provident abundance of resources}

After decades of scarcity, real and imaginary, and of regimes of funding that encouraged hyper-competition, the move to a research eco-system of abundance will necessitate new practical knowledge to navigate and optimize this. At some point, scientists will become comfortable knowing that they can find and demand what they need to do their work. They will become affluent academics: rich in the resources they require, even as they are detached from marketplace incentives. Like huntergatherers, their ability to glean what they need means they no long have any reason to hoard what they have. 
"In part, the Ju/'hoansi's affluence was based on their unyielding confidence in the providence of their environments and their skills at exploiting this. Ju/'hoansi still make use of well over 150 different plant species, and have the knowledge to hunt and trap pretty much any animal they choose to. As a result, they only ever worked to meet their immediate needs, did not store surpluses, and never harvested more than they could eat in the short term" (Suzman 2017 [The Guardian]).

Building provident academic resources (repositories, metadata, platforms, etc.) for reusable digital objects, global in scale but also localizable for use, is a major task for open science. The enormity of this task has occupied a lot of attention in the past decade. National and international data organizations, and professional associations and universities (with important nudges from funders) are working on parts of this task.

There is an equally important task that open scientists need to tackle: Governing these resources and forging the shared social practices that can do so. This second task represents a major cultural challenge for the open science academy, and for each part of this (academic departments, research labs, professional associations, universities, funding agencies); and, finally, for each individual open scientist. Culture is aways shared, and always carried by individuals.

Scarcity, as we will see next, is mainly manufactured to game values in the marketplace. If you make less of something, when the demand is constant, you an increase the price.

\section{Final thought: Abundance is how you perceive your work.}

"If you perceive the universe as being a universe of abundance then it will be. If you think of the universe as one of scarcity, then it will be. ... I always thought that there was enough of everything to go around-that there are enough ideas in the universe and enough nourishment." Milton Glaser in (Millman 2007).

\section{Scarcity is mostly manufactured and arbitrary}

"I am often told that I should be grateful for the progress that Western civilization has brought to these shores. I am not. This life of work-or-die is not an improvement on preinvasion living, which involved only a few hours of work a day for shelter and sustenance, performing tasks that people do now for leisure activities on their yearly vacations: fishing, collecting plants, hunting, camping, 
and so forth. The rest of the day was for fun, strengthening relationships, ritual and ceremony, cultural expression, intellectual pursuits, and the expert crafting of exceptional objects. I know this is true because I have lived like this, even in this era when the land is only a pale shadow of the abundance that once was. We have been lied to about the 'harsh survival' lifestyles of the past. There was nothing harsh about it. If it was so harsh-such a brutish, menial struggle for existencethen we would not have evolved to become the delicate, intelligent creatures that we are" (Yunkaporta 2020).

"As Lawrence Lessig has so persuasively argued over the years, there is nothing 'natural' about the artificial scarcity of intellectual property law. Those laws are deliberate interventions crafted by human intelligence and are enforced almost entirely by non-market powers. Jefferson's point, in his letter to McPherson, is that if you really want to get into a debate about which system is more 'natural,' then the free flow of ideas is always going to trump the artificial scarcity of patents. Ideas are intrinsically copyable in the way that food and fuel are not. You have to build dams to keep ideas from flowing" (Johnson 2011).

Ideas are not like food, nor fuel. But then the scarcity of jobs, careers, and funding is not simply artificial either. Scarcity is real, you might say. However real this scarcity is today, it is not necessarily as durable as you think. So, how does open science fuel a new abundance for these various resources?

Let's start with the problem. Scarcity is created to build markets where arbitrary values can be maintained to guarantee a profit. Widlock describes it this way: "Modernity defines itself as a 'culture of scarcity'..., of there never being enough goods and information. The assumed 'eternal shortage' paradoxically seems to grow, rather than to diminish, as modernity and capitalism unfold and ever more consumer needs are created. The underlying orthodox assumption is that economy is above all economizing (making ends meet) and directed toward utility as being conditioned by limited means employed to satisfy unlimited wants." (2016) Of course, the "wants" are also manipulated by the marketplace: they are attached to the outcomes of the scarcity machine.

Bauwens (2012) adds that markets cannot survive without scarcity: "Markets are defined as ways to allocate scarce resources, and capitalism is not just a scarcity 
'allocation' system, but, in reality, is a scarcity engineering system, which can only accumulate capital by constantly reproducing and expanding conditions of scarcity. When there is no tension between supply and demand, there can be no market, and so no capital accumulation" < http://realitysandwich.com/142773/evolving_partner_state_ethical_economy/> Accessed 10/2018). Markets only succeed when they can manufacture enough scarcity to raise the price of what they manufacture high enough to make a profit; they have the means to make enough of the goods to drive the price down, but chose not to do so (Siefkes 2008).

The market logic of scarcity, and the resulting neoliberal push to remake the academy as a business venture has been a uniform disaster for universities, and an existential threat to the reputation of science. Science findings that are infested with conflicts on interest create doubt in the public. When scientists are suspected of not being truthful, of not upholding the primary sincerity required for the scientific method to stand, then their findings, even when these might be true (to the amount this is available), will be met with doubt. This impacts the ability of the public to rely on science for their own sense-making, and undermines science's position in the public information ecology (See: The War on Sense-Making; Accessed 10/31/2020).

Open science alone cannot reverse this trend. Expecting open science to fix the academy without first extracting this from a hyper-rivalrous economy puts too much on the back of this cultural shift. But the practices of open science can offer expanded returns on the investments the state makes into the academy. These practices optimize the value of research objects and reground science by its internal norms. The culture of open science can help return the academy to a value proposition that centers research and teaching as a public good that reaches toward social justice and broadlyfelt benefits across society.

Open science values contributions from a broader cohort or intellectuals. Doctorate holders represent a tiny proportion of the planet's population (less than $2 \%$ ). But the problems the world faces are more complex and numerous than ever before. "There is, however, no limit on society's need to address complex challenges, the number of research questions that can be asked, or the amount of scientific work that can be done. New models are needed to help identify different ways for scientists to continue their work outside of a standard academic or agency job” (Lancaster, et al. 2018). 


\section{Right now is the time to euthanize Matthew}

Open science culture prioritizes widespread, global participation, which would necessitate redistributions of academic fortunes away from current elite organizations and funder-favorite research endeavors: the result of decades of cumulative advantages, sometimes called the "Matthew Effect." The process of "un-accumulating" advantages in the academy will be painful, particularly to those who have invested in them.

Today, "[d]ifferences in individual capabilities aside, then, processes of cumulative advantage and disadvantage accentuate inequalities in science and learning: inequalities of peer recognition, inequalities of access to resources, and inequalities of scientific productivity" (Merton 1988). Tomorrow, an open-science academy will need to refactor decision processes to spread around resources that are currently limited, and challenge the current level of available funding by making the case that it can optimize the return on the value-including a wide range of social benefits-for public investment in the public goods of science.

"The ecosystem metaphor is our attempt to reframe and expand the discussion of STEM careers and science beyond what has often become a sterile and arid debate about competition and scarcity within academia by connecting it with the open flows, resilence, diversity and feed-back loops of ecological systems. ...[W]e strongly believe that making science better is not just 'making the incentives better', but a collective cultural shift beyond viewing competition and individualistic success as the sole defining feature of science (i.e., the pipeline model)" (Lancaster et al. 2018).

Instead of an economy that relies on hyper-competition to create an underclass of PhDs (Chapman, C.A., et al. 2019) for hire at almost any price, an open, sharing academy economy would find guaranteed employment for all PhDs at one of the many available academy institutions, or elsewhere in society (See: Tcherneva (2020) on the benefits of job guarantees).

\section{Bibliography: Oppen Scientist Handbook References}

